\title{
ON NONCOMMUTATIVE VNL-RINGS AND GVNL-RINGS
}

\author{
WEIXING CHEN \\ School of Mathematics and Information Science, Shandong Institute of Business and \\ Technology, Yantai 264005, P.R. China \\ e-mail: wxchen5888@163.com \\ and WENTING TONG \\ Department of Mathematics, Nanjing University, Nanjing 210093, P.R. China \\ e-mail:wttong@nju.edu.cn
}

(Received 12 January, 2005; accepted 16 September, 2005)

\begin{abstract}
It is proved that every abelian VNL-ring is an SVNL-ring, which gives a positive answer to a question of Osba et al. [7]. Some characterizations of duo VNLrings are given and some main results of Osba et al. [7] on commutative VNL-rings are extended to right duo VNL-rings and even abelian GVNL-rings.
\end{abstract}

2000 Mathematics Subject Classification. 16D25, 16E50.

1. Introduction. Rings considered are associative with identity unless the contrary is stated explicitly. An element $a$ of a ring $R$ is $\pi$-regular if there exist a positive integer $n$ and $x \in R$ such that $a^{n}=a^{n} x a^{n}$. In the case of $n=1, a$ is regular. A ring $R$ is $\pi$-regular (regular) if every element in $R$ is $\pi$-regular (regular). A ring $R$ is an exchange ring if for every $a \in R$ there exists an idempotent $e \in R$ such that $e \in a R$ and $(1-e) \in(1-a) R$. A ring $R$ is right (left) duo if every right (left) ideal is two-sided, and $R$ is $d u o$ if $R$ is right and left duo. A ring $R$ is abelian if all idempotents are contained in the center and a ring $R$ is reduced if it does not contain nonzero nilpotent elements.

Following Contessa [4], a commutative ring $R$ is a $V N L$-ring if for every $a \in R$, at least one of $a$ or $1-a$ is regular. According to Osba et al. [7], a commutative ring $R$ is an $S V N L$-ring if whenever $(S)=R$ for some nonempty subset $S$ of $R$, at least one of the elements in $S$ is regular, where $(S)$ is an ideal generated by $S$. Some properties of VNL-rings and SVNL-rings are investigated in Osba et al. [7]. But they are unable to solve the question whether every VNL-ring is an SVNL-ring. Because of this they are unable to characterize VNL-rings abstractly in the sense of relating them to more familiar classes of rings. In the present paper we define a noncommutative ring $R$ to be a $V N L$-ring ( $G V N L$-ring) if for every $a \in R$ at least one of $a$ or $1-a$ is regular ( $\pi$-regular) and a ring $R$ is an SVNL-ring, if whenever $(S)_{r}=R$ for some nonempty subset $S$ of $R$, at least one element in $S$ is regular, where $(S)_{r}$ is a right ideal generated by $S$. The main purpose of this paper is to prove that every abelian VNL-ring is an SVNL-ring, giving an answer to the question of Osba et al. [7] in the affirmative. Furthermore we give some characterizations of duo VNL-rings, and extend and improve some main results of Osba et al. [7] on commutative VNL-rings.

Throughout this paper we use the symbol $J(R)$ to denote the Jacobson radical of a ring $R, \operatorname{Id}(R)$ its set of idempotents, and $\operatorname{Max}(R)$ its maximal spectrum. The left annihilator of an element $a$ in $R$ is denoted by $A_{l}(a)$. For an integer $n \geq 2$, the symbol 
$\mathbb{Z}_{n}$ stands for the ring of integers modulo $n$. Also for two sets $A$ and $B$ with $B \subseteq A$, the symbol $A \backslash B$ or $A-B$ denotes the supplementary set of $B$ in $A$.

2. Abelian VNL-rings. We start this section with the following definition.

Definition 2.1. An element $a$ of a ring $R$ is called an exchange element if there exists an idempotent $e \in R$ such that $e \in a R$ and $1-e \in(1-a) R$.

Obviously, a ring $R$ is an exchange ring if and only if every element of $R$ is an exchange element. And an element $a$ of a ring $R$ is an exchange element if and only if so is $1-a$. It is known from Nicholsion ([6], Proposition 1.6) or Stock ([8], Example 2.3 ) that a regular element ( $\pi$-regular element) of a ring $R$ is an exchange element. So we have the following.

THEOREM 2.2. Every VNL-ring (GVNL-ring) is an exchange ring.

Recall that an ideal $I$ in a ring $R$ is regular if for each $a \in I$, there exists $x \in I$ such that $a=\operatorname{axa}$ (Goodearl, [5], $P_{2}$ ).

The following facts are essential to obtaining our main results.

Lemma 2.3 (Goodearl, [5], Proposition 1.5). Let $R$ be a ring, and set $M(R)=\{x \in$ $R \mid R x R$ is a regular ideal\}. Then the following hold:

(a) $M(R)$ is a regular ideal of $R$.

(b) $M(R)$ contains all regular ideals of $R$.

(c) $R / M(R)$ has no nonzero regular ideal.

LEMMA 2.4 (Goodearl, [5], $P_{2}$ ). Let $R$ be a ring. If $x, y \in R$ and $x^{\prime}=x-x y x$, and if $x^{\prime}=x^{\prime} a x^{\prime}$ for some $a \in R$, then $x=x b x$ for some $b \in R$.

Lemma 2.5 (Warfield, [9], Theorem 1). An exchange ring with only two idempotents is a local ring.

LEMmA 2.6. Let $R=\prod_{\alpha \in \Lambda} R_{\alpha}$ be a ring. Then $R$ is a VNL-ring (abelian VNL-ring) if and only if there exists $\alpha_{0} \in \Lambda$ such that $R_{\alpha_{0}}$ is a VNL-ring (abelian VNL-ring) and for each $\alpha \in \Lambda-\left\{\alpha_{0}\right\}, R_{\alpha}$ is a regular (abelian regular) ring.

Proof. It is very similar to that of Osba et al. ([4], Theorem 3.1).

LEMMA 2.7. Let $R$ be an abelian $V N L$-ring and not regular. Then $R / M(R)$ is a local ring, where the meaning of $M(R)$ is the same as that in Lemma 2.3.

Proof. It is known and easy to prove that the homomorphic image of a VNL-ring is a VNL-ring. Since $R$ is a VNL-ring, $R / M(R)$ is a VNL-ring and so is an exchange ring by Theorem 2.2. Let $\bar{R}=R / M(R)$. If $R / M(R)$ is not a local ring, then by Lemma 2.5 there exists a nontrivial idempotent $\bar{e} \in \bar{R}$. And hence $\bar{R}=\bar{e} \bar{R} \bigoplus(\overline{1}-\bar{e}) \bar{R}$. Since $R$ is an abelian exchange ring, the idempotents of $R / M(R)$ can be lifted to $R$, so $R / M(R)$ is an abelian exchange ring and hence an abelian VNL-ring. Now Lemma 2.6 implies that at least one of $\bar{e} \bar{R}$ or $(\overline{1}-\bar{e}) \bar{R}$ is an abelian regular ring, so is a nonzero regular ideal of $\bar{R}$, which contradicts Lemma 2.3. And the proof is completed.

THEOREM 2.8. Let $R$ be an abelian ring. Then $R$ is a VNL-ring if and only if it is an SVNL-ring.

Proof. $(\Rightarrow)$ If $R$ is regular, then we are done. Otherwise $R$ is a VNL-ring which is not regular. Now for any nonempty subset $S$ of $R$ with $(S)_{r}=R$, there exist 
$s_{1}, s_{2}, \ldots, s_{n} \in S$ such that $s_{1} R+s_{2} R+\cdots+s_{n} R=R$, hence there exist $r_{1}, r_{2}, \ldots, r_{n} \in$ $R$ satisfying $s_{1} r_{1}+s_{2} r_{2}+\cdots+s_{n} r_{n}=1$ and so $\overline{s_{1}} \overline{r_{1}}+\overline{s_{2}} \overline{r_{2}}+\cdots+\overline{s_{n}} \overline{r_{n}}=\overline{1}$ in $\bar{R}=$ $R / M(R)$. According to Lemma $2.7, \bar{R}$ is a local ring. It follows that not all $\overline{s_{i}}$ are in $J(\bar{R})$. So there exists an $\overline{s_{k}}$ such that $\overline{s_{k}}$ is a unit in $\bar{R}$, hence $\overline{s_{k}}$ is a regular element in $\bar{R}$. Assume that $\overline{s_{k}}=\overline{s_{k}} \overline{x_{k}} \overline{s_{k}}$ for some $\overline{x_{k}} \in \bar{R}$. Then we have $s_{k}-s_{k} x_{k} s_{k} \in M(R)$ and $s_{k}-s_{k} x_{k} s_{k}=\left(s_{k}-s_{k} x_{k} s_{k}\right) y_{k}\left(s_{k}-s_{k} x_{k} s_{k}\right)$ for some $y_{k} \in R$, thus $s_{k}$ is a regular element by Lemma 2.4 and so $R$ is an SVNL-ring.

$(\Leftarrow)$ Assume that $R$ is an SVNL-ring. For any $a \in R$, let $S=\{a, 1-a\}$. Then $(S)_{r}=R$ since $1=a+1-a \in(S)_{r}$. Hence either $a$ or $1-a$ is regular and $R$ is a VNL-ring.

COROLlaRY 2.9. Let $R$ be a commutative ring. Then $R$ is a VNL-ring if and only if it is an SVNL-ring.

The above corollary gives a positive answer to the question whether every commutative VNL-ring is an SVNL-ring.

Although the following corollary is observed by Osba et al. [5], its proof may not be trivial until we obtain the above theorem.

COROLLARY 2.10. The homomorphic image of a commutative (abelian) SVNL-ring is a commutative (abelian) SVNL-ring.

Proof. Since the homomorphic image of a commutative (abelian) VNL-ring is a commutative (abelian) VNL-ring, we get the desired conclusion by Theorem 2.8.

Next extend some main results of Osba et al. [4] on commutative VNL-rings to right duo VNL-rings and we give some characterizations of duo VNL-rings.

Recall that an ideal $I$ of a ring $R$ is (left) pure if $I=m I$, where $m I=\{a \in R \mid a=i a$ for some $i \in I\}$. It is easy to check that for a right duo ring $R$ and any $a \in R, A_{l}(a)$ is a two-sided ideal and $m I=\left\{a \in R \mid I+A_{l}(a)=R\right\}$.

LEMMA 2.11. Let $R$ be a right duo ring and $a \in R$. Then $a R$ is pure if and only if $a R+A_{l}(a)=R$.

Proof. Suppose that $a R$ is pure. Then there exists $x=a r \in a R$ such that $a=x a=$ ara. So $(1-a r) a=0$ which implies that $1-a r \in A_{l}(a)$. Hence $1=a r+(1-a r) \in$ $a R+A_{l}(a)$, and $a R+A_{l}(a)=R$. Conversely, assume that $a R+A_{l}(a)=R$. Then there exist $r \in R, b \in A_{l}(a)$ such that $a r+b=1$. So $a r a+b a=a$, i.e., $a=$ ara since $b a=0$. So for any $x \in R$, we have $a x=\operatorname{arax}$ with $a r \in a R$, which implies that $a R$ is pure.

THEOREM 2.12. Let $R$ be a right duo ring and $a \in R$. Then $a$ is regular if and only if for every maximal ideal $M, a \in M$ implies $a \in m M$.

Proof. Suppose that $a$ is regular. If $M \in \operatorname{Max}(R)$ is such that $a \in M$, then $a=$ ara for some $r \in R$ with $a r \in M$ and hence $a \in m M$. Conversely, assume that for each maximal ideal $M, a \in M$ implies $a \in m M$. We claim that $a R+A_{l}(a)=R$. If not, there exists a maximal ideal $M$ such that $a R+A_{l}(a) \subseteq M$. Note that $a \in M$ implies $a \in m M$. There exists $x \in M$ such that $a=x a$. So $(1-x) a=0$, which gives $1-x \in A_{l}(a) \subseteq M$. Hence $1 \in M$, a contradiction. Thus $a R+A_{l}(a)=R$, there exist $r \in R$ and $c \in A_{l}(a)$ such that $a r+c=1$, which implies $a=\operatorname{ar} a$ and $a$ is regular.

Corollary 2.13. A right duo ring $R$ is regular if and only if all maximal ideals are pure. 
Proof. If $R$ is regular, then for every $M \in \operatorname{Max}(R), a \in M$ implies $a \in m M$ by Theorem 2.12, which gives $m M=M$ and hence $M$ is pure. Conversely, if every $M \in$ $\operatorname{Max}(R)$ is pure, then $M=m M$ and so $a \in M$ implies $a \in m M$ for every $a \in R$. Hence for every $a \in R, a$ is regular by Theorem 2.12 and so $R$ is regular.

THEOREM 2.14. The following statements are equivalent for a duo ring $R$.

(a) All maximal ideals of $R$ except may be one are pure.

(b) There exists $N \in \operatorname{Max}(R)$ such that for each a $\notin N$, a is regular.

(c) The ring $R$ is a VNL-ring.

Proof. (a) $\Rightarrow$ (b) Suppose that there exists $N \in \operatorname{Max}(R)$ such that $M=m M$ for each $M \in \operatorname{Max}(R) \backslash\{N\}$. If $a \notin N$, then for each $M \in \operatorname{Max}(R), a \in M$ implies $M \neq N$, so $M=m M$ and $a \in m M$. Hence $a$ is regular by Theorem 2.12 .

(b) $\Rightarrow$ (c) Assume that there exists $N \in \operatorname{Max}(R)$ such that for each $a \notin N, a$ is regular. For each $a \in R$, if $a \notin N$, then we are done. Otherwise $a \in N$, so $1-a \notin N$ and hence $1-a$ is regular.

(c) $\Rightarrow$ (b) First note that a duo ring is abelian. Now suppose that $R$ is a VNL-ring. Then it is an SVNL-ring by Theorem 2.8. Let $T$ be the set of elements which are not regular in $R$. If $T$ is empty, then we are done. Otherwise consider the right ideal $I$ generated by $T$, which is an ideal since $R$ is duo. If $1 \in I$, then $1=\sum_{i=1}^{n} s_{i} r_{i}$ with $s_{i} \in T$ and $r_{i} \in R$ for each $i$. Thus $R=s_{1} R+s_{2} R+\cdots+s_{n} R$ and there exists $i$ such that $s_{i} \notin T$, a contradiction. Hence $I$ is contained in a maximal $N$ and for each $a \notin N$, $a$ is regular.

(b) $\Rightarrow$ (a) Assume that there exists $N \in \operatorname{Max}(R)$ such that for each $a \notin N, a$ is regular. Let $M \in \operatorname{Max}(R) \backslash\{N\}$ and $a \in M$. If $a \notin N$, then $a$ is regular and so $a \in m M$ by Theorem 2.12. If $a \in M \bigcap N$, then choose $b \in M \backslash N$, so that $b \in m M$. Clearly $a+b \in M \backslash N$, which implies $a+b \in m M$. We need to prove $a \in m M$. Since $b \in m M$, $M+A_{l}(b)=R$ and there exist $m_{1} \in M, x_{1} \in A_{l}(b)$ such that $m_{1}+x_{1}=1$. (*) Similarly, $a+b \in m M$ implies $M+A_{l}(a+b)=R$ and there exist $m_{2} \in M, x_{2} \in A_{l}(a+b)$ such that $m_{2}+x_{2}=1$. (**) Equations $(*)$ and $(* *)$ imply $m_{3}+x_{2} x_{1}=1$ with $m_{3}=m_{2} m_{1}+$ $m_{2} x_{1}+x_{2} m_{1} \in M$. Since $R$ is duo,we have $x_{2} x_{1}=y_{1} x_{2}$ for some $y_{1} \in R$. It follows that $x_{2} x_{1}(a+b)=0$ and $x_{2} x_{1} b=0$, which implies $x_{2} x_{1} a=0$. Hence $M+A_{l}(a)=R$ and so $a \in m M$.

The following corollary characterizes a commutative VNL-ring, and the equivalence of (a) and (b) is known in Osba et al. [4].

COROLLARY 2.15. The following are equivalent for a commutative ring $R$.

(a) All maximal ideals of $R$ except may be one are pure.

(b) There exists $N \in \operatorname{Max}(R)$ such that for each $a \notin N$, a is regular.

(c) The ring $R$ is a VNL-ring.

3. Abelian GVNL-rings. In this section we study abelian GVNL-rings, extending some main results of Osba et al. [4] on commutative VNL-rings to abelian GVNL-rings.

EXAMPLE 3.1. There is a commutative GVNL-ring $R$ which is not a VNL-ring.

Proof. Let $R=\mathbb{Z}_{4} \bigoplus \mathbb{Z}_{4}$. Then it is easy to check that $(\overline{3}, \overline{2})$ and $(\overline{1}, \overline{1})-(\overline{3}, \overline{2})$ are not regular, so $R$ is not a VNL-ring. Since $R$ is $\pi$-regular, it is a GVNL-ring.

In the above example $R$ satisfies $J(R) \neq 0$. We naturally ask whether there exists an abelian GVNL-ring $R$ such that $J(R)=0$ but it is not a VNL-ring. The following proposition shows that the answer is negative. 
Proposition 3.2. If $R$ is an abelian $G V N L$-ring with $J(R)=0$, then $R$ is a $V N L$-ring.

Proof. It is known by Lemma 4.10 in (Stock, [8]) that for an exchange ring $R$ with $J(R)=0, R$ is abelian if and only if it is reduced. Now $R$ is a reduced exchange ring by Theorem 2.2. Now for any $a \in R$, if $a$ is $\pi$-regular, then $a^{n}=a^{n} x a^{n}$ for some positive integer $n$ and $x \in R$. Clearly, $e=a^{n} x \in \operatorname{Id}(R)$. So $((1-e) a)^{n}=$ $(1-e) a^{n}=(1-e) e a^{n}=0$, and hence $(1-e) a=0$. Therefore $a=e a=a\left(a^{n-1} x\right) a$ is regular. And the proof is completed.

Since the homomorphic image of a (an abelian) GVNL-ring is also a (an abelian) GVNL-ring, the following corollary is immediate.

COROLlary 3.3. If $R$ is an abelian $G V N L$-ring, then $R / J(R)$ is an abelian $V N L$-ring.

LeMmA 3.4. Let $R=\bigoplus_{i=1}^{k} R_{i}$ be an abelian ring. Then $\left(a_{1}, a_{2}, \ldots, a_{k}\right)$ is $\pi$-regular in $R$ if and only if every $a_{i}$ is $\pi$-regular in $R_{i}$.

Proof. It is sufficient to prove the case of $k=2$. Clearly $R$ is abelian if and only if every $R_{i}$ is abelian. Now suppose that $a_{1}$ and $a_{2}$ are $\pi$-regular. Then there exist positive integers $m, n$ and $x, y \in R$ such that $a_{1}^{m}=a_{1}^{m} x a_{1}^{m}$ and $a_{2}^{n}=a_{2}^{n} y a_{2}^{n}$. Since $x a_{1}^{m}$, and $y a_{2}^{n}$ are idempotents, we have $a_{1}^{m n}=a_{1}^{m n}\left(x a_{1}^{m}\right)^{n}=a_{1}^{m n}\left(x a_{1}^{m}\right) \cdots\left(x a_{1}^{m}\right)=$ $a_{1}^{m n} x a_{1}^{m}\left(x a_{1}^{m}\right)\left(x a_{1}^{m}\right) \cdots\left(x a_{1}^{m}\right)=a_{1}^{m n} x^{2} a_{1}^{m}\left(x a_{1}^{m}\right)\left(x a_{1}^{m}\right) \cdots\left(x a_{1}^{m}\right) a_{1}^{m}=a_{1}^{m n} x^{3} a_{1}^{m}\left(x a_{1}^{m}\right)\left(x a_{1}^{m}\right) \cdots$ $\left(x a_{1}^{m}\right) a_{1}^{2 m}=a_{1}^{m n} x^{n} a_{1}^{m n}$. Similarly, $a_{2}^{m n}=a_{2}^{m n} y^{m} a_{2}^{m n}$ and so $\left(a_{1}, a_{2}\right)^{m n}=\left(a_{1}^{m n}, a_{2}^{m n}\right)=$ $\left(a_{1}^{m n} x^{n} a_{1}^{m n}, a_{2}^{m n} y^{m} a_{2}^{m n}\right)=\left(a_{1}^{m n}, a_{2}^{m n}\right)\left(x^{n}, y^{m}\right)\left(a_{1}^{m n}, a_{2}^{m n}\right)=\left(a_{1}, a_{2}\right)^{m n} \quad\left(x^{n}, y^{m}\right) \quad\left(a_{1}, a_{2}\right)^{m n}$. Hence $\left(a_{1}, a_{2}\right)$ is $\pi$-regular. Using induction on $k$, we can obtain the desired conclusion. Conversely, if $\left(a_{1}, a_{2}, \ldots, a_{k}\right)$ is $\pi$-regular in $R$, then obviously every $a_{i}$ is $\pi$-regular in $R_{i}$.

COROLlary 3.5. Let $R=\bigoplus_{i=1}^{n} R_{i}$ be a ring. Then $R$ is abelian $\pi$-regular if and only if every $R_{i}$ is abelian $\pi$-regular.

THEOREM 3.6. Let $R=\bigoplus_{i=1}^{n} R_{i}$ be a ring. Then $R$ is an abelian $G V N L$-ring if and only if there exists an index $k$ such that $R_{k}$ is a GVNL-ring and for each $i \neq k, R_{i}$ is a $\pi$-regular ring.

Proof. Suppose that $R$ is GVNL-ring. Then $R_{i}$ is a GVNL-ring as a homomorphic image of $R$ for each $i$. If every $R_{i}$ is a $\pi$-regular ring, then we are done. Otherwise, there exists an index $k$ such that $R_{k}$ is not a $\pi$-regular ring. Assume that $a_{k} \in R_{k}$ is not $\pi$-regular. Then for each $i \neq k$ and any $a_{i} \in R_{i},\left(1-a_{1}, \ldots, 1-\right.$ $\left.a_{k-1}, a_{k}, 1-a_{k+1}, \ldots, 1-a_{n}\right)$ is not $\pi$-regular by Lemma 3.4. Thus $\left(a_{1}, \ldots, a_{k-1}, 1-\right.$ $\left.a_{k}, a_{k+1}, \ldots, a_{n}\right)$ is $\pi$-regular, and so is every $a_{i}$ whenever $i \neq k$. Therefore $R_{i}$ is $\pi$ regular for each $i \neq k$.

Conversely, assume that that there exists an index $k$ such that $R_{k}$ is a GVNLring and, for each $i \neq k, R_{i}$ is $\pi$ regular. We prove that $R$ is a GVNL-ring. For each $a=\left(a_{1}, \ldots, a_{k-1}, a_{k}, a_{k+1}, \ldots, a_{n}\right) \in R$, if $a_{k}$ is $\pi$-regular, then $a$ is $\pi$-regular by Lemma 3.4. If $a_{k}$ is not $\pi$-regular, then $1-a_{k}$ is $\pi$-regular, so is $1-a$. And the proof is completed.

THEOREM 3.7. Let $R$ be an abelian GVNL-ring in which 2 is a unit. Then every element in $R$ is a sum of a unit and a square root of 1 (i.e., an element a with $a^{2}=1$ ).

Proof. Suppose that $R$ is a GVNL-ring. By Theorem 2.2, $R$ is an exchange ring. Since $R$ is abelian, it is a clean ring by Nicholson ([6], Proposition 1.8). According to 
Camillo and $\mathrm{Yu}$ ([3], Theorem 11), every element of a clean ring in which 2 is invertible is a sum of a unit and a square root of 1 and so we are done.

COROLlary 3.8 (Osba et al. 2004, Theorem 3.8). Every element of a commutative $V N L$-ring in which 2 is a unit is a sum of no more than three units.

THEOREM 3.9. Let $R$ be an abelian ring with only a finite number of idempotents. Then $R$ is a GVNL-ring if and only if it is the direct product of a local ring and finitely many $\pi$-regular local rings each of which has the the property that the Jacobson radical coincides its set of nilpotent elements.

Proof. Suppose that $R$ is an abelian GVNL-ring. Then it is an exchange ring by Theorem 2.2. According to Camillo and Yu ([3], Theorem 9), $R$ is a semiperfect ring. There exist $e_{1}, e_{2}, \ldots, e_{n} \in I d(R)$ such that $e_{1}+e_{2}+\cdots+e_{n}=1$ and $e_{i} s$ are mutually orthogonal local idempotents. It follows that $R=e_{1} R \bigoplus e_{2} R \bigoplus \cdots \bigoplus e_{n} R$, where $e_{i} R$ is a local ring for each $i$. Now Theorem 3.6 implies that there exists $k$ such that $e_{k} R$ is a GVNL-ring and $e_{j} R$ is a $\pi$-regular ring for each $j \neq k$. By Badawi ([2], Lemma 5), we know that $J\left(e_{j} R\right)$ coincides with the set of nilpotent elements in $e_{j} R$. Conversely, if $R$ is the direct product of a local ring and finitely many $\pi$-regular local rings, then it is a GVNL-ring by Theorem 3.6.

COROLLARY 3.10. Let $R$ be an abelian ring with only a finite number of idempotents. Then $R$ is a VNL-ring if and only if it is the direct product of finitely many division rings and a local ring.

Proof. By Lemma 2.6 and the fact that a local regular ring is a division ring.

In the case of $R$ is a commutative ring, we have the following corollary.

COROLlaRY 3.11 (Osba, [4], Theorem 6.1). If $R$ is a commutative VNL-ring with only a finite number of idempotents. Then it is the direct product of finitely many regular rings and a local ring.

According to Ara [1], a ring $I$ (without unit) is called an exchange ring if for each $a \in I$ there exist an idempotent $e \in I$ and $r, s \in I$ such that $e=a r=a+s-a s$. Also if $I$ is an ideal of a unital exchange ring, then $I$ satisfies the above condition.

LEMma 3.12. Let $R$ be a ring. Then $R[x]$ is not an exchange ring.

Proof. Suppose that $R[x]$ is an exchange ring. Then for $x \in R[x]$ there exist an idempotent $e(x) \in R[x]$ and $r(x), s(x) \in R[x]$ such that $e(x)=x r(x)=x+s(x)-x s(x)$. Thus $e(x)=a_{1} x+a_{2} x^{2}+\cdots+a_{n} x^{n}$, and hence $e(x)=0$ by a direct calculation, so that $x+s(x)=x s(x)$, which is impossible by comparing the coefficients.

COROLlaRY 3.13. For any ring $R$, the ring $R[x]$ is not a $G V N L$-ring.

COROLlary 3.14 (Osba et al., [4], Corollary 4.8). For a commutative ring $R$, the ring $R[x]$ is not a $V N L$-ring.

THEOREM 3.15. The following statements are equivalent for an abelian ring $R$.

(a) The ring $R$ is a local ring.

(b) The ring $R[[x]]$ is a local ring.

(c) The ring $R[[x]]$ is a GVNL-ring.

(d) The ring $R$ is a GVNL-ring and $\operatorname{Id}(R)=\{0,1\}$. 
Proof. $(a) \Rightarrow(b)$ is known.

$(b) \Rightarrow(c)$ Since a local ring is a VNL-ring, it is a GVNL-ring.

$(c) \Rightarrow(d)$ Since $R$ is a homomorphic image of $R[[x]], R$ is a GVNL-ring. If $\operatorname{Id}(R) \neq$ $\{0,1\}$, then there exists a nontrivial idempotent $e \in R$. Hence $R=e R \bigoplus(1-e) R$ and $R[[x]]=e R[[x]] \bigoplus(1-e) R[[x]]$. By Theorem 3.6, $e R[[x]]$ or $(1-e) R[[x]]$ is $\pi$ regular, say for example $e R[[x]]$. Then $(e x)^{n}=(e x)^{n} f(x)(e x)^{n}$ for some $f(x) \in R[[x]]$ and some positive integer $n$. This implies $e=0$ by comparing the coefficients, which is a contradiction.

$(d) \Rightarrow(a)$ Since $R$ is abelian, $\operatorname{Id}(R)=I d(R[[x]])$. Hence $R$ is an exchange ring with only two idempotents and so $R$ is a local ring.

Combining Theorem 2.8 with Theorem 3.15, we have the following corollary.

Corollary 3.16 (Osba, [4], Theorem 4.6). For a commutative ring $R$, the following statements are equivalent.

(a) The ring $R$ is a local ring.

(b) The ring $R[[x]]$ is a local ring.

(c) The ring $R[[x]]$ is an $S V N L$-ring.

(d) The ring $R[[x]]$ is a $V N L$-ring.

(e) The ring $R$ is an $S V N L$-ring and $\operatorname{Id}(R)=\{0,1\}$.

(f) The ring $R$ is a $V N L$-ring and $I d(R)=\{0,1\}$.

(g) For each $a \in R$, a is a unit or $1-a$ is a unit.

We conclude this paper with the following open question:

QUESTION 3.17. Is every noncommutative VNL-ring an SVNL-ring?

ACKNOWLEDGEMENTS. This research was partially supported by the Doctorate Foundation of China Education Ministry (Grant No. 20020284009). Also the authors wish to express their gratitude to the referee for valuable comments.

\section{REFERENCES}

1. P. Ara, Extensions of exchange rings, J. Algebra 197 (1997), 409-423.

2. A. Badawi, On abelian $\pi$-regular rings, Comm. Algebra 25(4) (1997), 1009-1021.

3. V. P. Camillo and H. P. Yu, Exchange ring, units and idempotents, Comm. Algebra 22(12) (1994), 4737-4749.

4. M. Contessa, On certain classes of PM-rings, Comm. Algebra 12 (1984), 1447-1469.

5. K. R. Goodearl, Von Neumann regular rings (Pitman, London, 1979).

6. W. K. Nicholson, Lifting idempotents and exchange rings, Trans. Amer. Math. Soc. 229 (1977), 269-278.

7. E. A. Osba, M. Henriksen and O. Alkam, Combining local and von Neumann regular rings, Comm. Algebra 32(7) (2004), 2639-2653.

8. J. Stock, On rings whose projective modules have the exchange property, J. Algebra 103 (1986), 437-453.

9. R. B. Warfield, A Krull Schmidt theorem for infinite sums of modules, Proc. Amer. Math. Soc. 22 (1969), 460-465. 